\title{
Heralding on the Detection of Zero Photons
}

\author{
C.M. Nunn, J.D. Franson, and T.B. Pittman \\ Department of Physics, University of Maryland Baltimore County, Baltimore, MD 21250, USA
}

(Dated: October 1, 2021)

\begin{abstract}
Although heralding signals in quantum optics experiments are typically based on the detection of exactly one photon, it has recently been theoretically shown that heralding based on the detection of zero photons can be useful in a number of quantum information applications. Here we experimentally investigate a technique for "heralding on zero photons" using conventional single-photon detectors. We illustrate how detector efficiency and dark count rates play a counterintuitive role in the ability to accurately detect zero photons, and use a variant of the Hong-Ou-Mandel interferometer to study the deleterious effects of limited detector efficiency when heralding on zero.
\end{abstract}

\section{INTRODUCTION}

Conditional measurements have a long and fruitful history in quantum optics [1] and quantum state engineering experiments [2]. The process often involves systems in which the detection of a single photon in one output mode is used to probabilistically herald the presence of a desired quantum state in a different output mode. A particularly powerful early example is the realization of a single-photon source based on Parametric Down Conversion (PDC) [3. There, the detection of one member of a randomly emitted photon pair is used to herald the presence of the twin photon, which can then be used for subsequent applications [4, 5. More complex heralding examples include ideas ranging from photon subtraction [6] and quantum scissors [7, to the realization of probabilistic entangling gates in the Linear Optics Quantum Computing (LOQC) paradigm [8]. Many of these applications have benefited from the recent advances in the development of photon-number resolving (PNR) detectors [9] which enable heralding signals based on the detection of exactly 2,3 , or even larger numbers of photons [10, 11].

Somewhat surprisingly, heralding signals based on the detection of zero photons are also useful. For example, "heralding on zero" is an essential feature of LOQC [8], and forms the basis of noiseless attenuation for quantum communications 12 14. Figure 1 provides a simplified overview of the operational principle within these contexts. Here, an input state $|\psi\rangle_{\text {in }}$ enters a system that contains, for example, a carefully designed interferometric device with two output ports. In panel (a), the device is designed in such a way that the detection of exactly 1 photon in the upper output mode (a "click" event) heralds the presence of the desired state $|\psi\rangle_{\text {out }}$ in the other output mode. In panel (b), the device is designed so that the detection of exactly zero photons (a "no-click" event) heralds a different desired output state $\left|\psi^{\prime}\right\rangle_{\text {out }}$.

The core idea is that regardless of photon number, conditioning on the detection of a Fock state $|n\rangle$ in one mode can be a powerful tool for quantum state engineering [2]. Although extending this idea to $n=0$ is straightforward in theory, experimentally detecting zero photons presents a number of challenges [15 17] and motivates

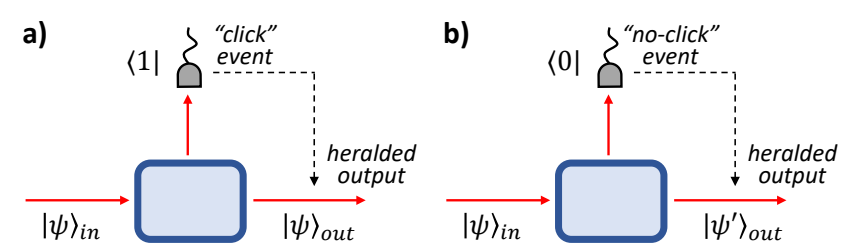

FIG. 1. Comparison of quantum state engineering via (a) conventional heralding on the detection of exactly 1 photon, and (b) heralding on the detection of exactly zero photons. In both cases, a unique detection signal (a "click" or "no-click" event) in one output mode is used to probabilistically herald the presence of a desired output state in the other output mode.

the need for effective "heralding on zero" techniques [1824. In this paper, we explore the use of standard singlephoton counting modules (SPCMs) for this unique application. In comparison with conventional heralding techniques based on the detection of 1 (or more) photons, the problematic roles of detector inefficiency and dark counts in realistic detectors are reversed. We begin by highlighting this idea with a simplified gedankenexperiment, and then experimentally demonstrate the effects through a unique signal that arises when "heralding on zero" in an otherwise conventional Hong-Ou-Mandel (HOM) interferometer 25] fed with photon pairs from a pulsed PDC source.

\section{OVERVIEW}

To illustrate the basic technique of detecting zero photons with conventional single-photon detectors, we consider the simple gedankenexperiment experiment shown in Figure 2a. An input Fock state $|1\rangle$ is prepared with an idealized pulsed single-photon source (SPS), then sent into a 50/50 beamsplitter (BS). This single optical element serves as the general interferometric device first shown in Figure 1b (the blue box). Reflected photons from the beamsplitter are monitored with a single-photon detector $D_{1}$. Additionally, each time the SPS emits a single photon, it also emits a strong optical reference pulse that is detected by an auxiliary reference detector $D_{\text {ref }}$ 


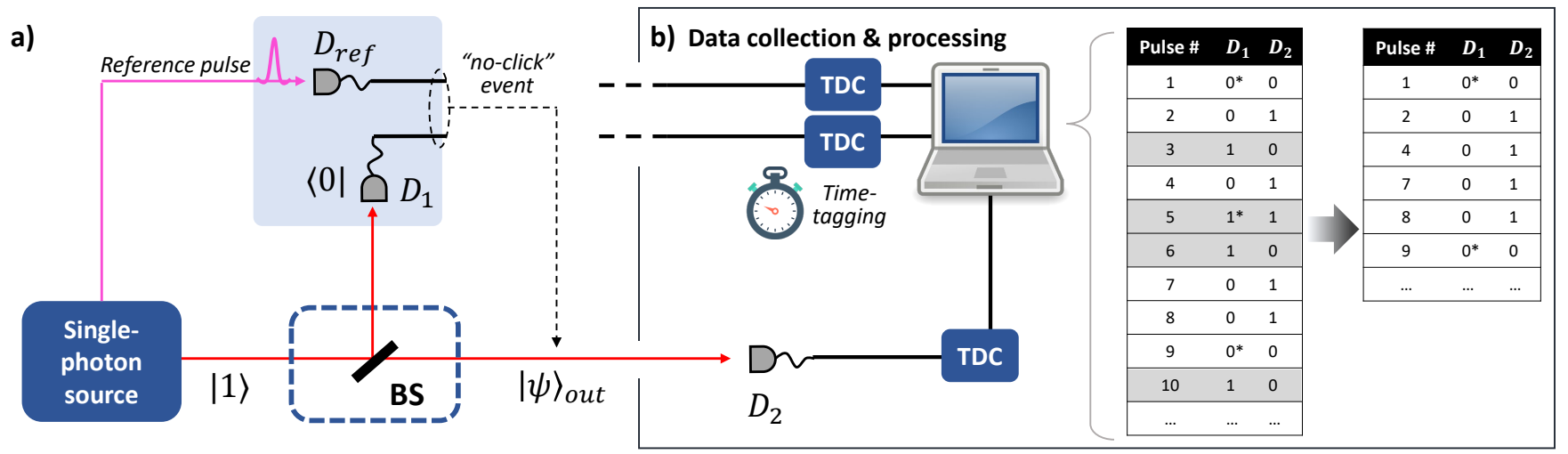

FIG. 2. (a) Simple gedankenexperiment using a pulsed single-photon source and 50/50 beamsplitter (BS) to highlight the role of detector inefficiency and dark counts when heralding on zero. Here, a detection at $D_{r e f}$ combined with the absence of a detection signal from $D_{1}$ results in a "no-click" event, ideally heralding a single photon in the output. (b) Visualization of the measurement process used to verify the outcome in a realistic experimental setup using an auxiliary detector $D_{2}$, and time-to-digital converters (TDCs) for data collection and post-processing. $D_{1}$ dark counts and inefficiency errors are marked with asterisks, and negatively impact the heralded output state.

with perfect efficiency. As shown in the shaded box of Fig. 2(a), the detection of zero photons simply corresponds to a detection by $D_{\text {ref }}$, and the simultaneous absence of a detection by detector $D_{1}$. Crucially, the unaccompanied reference pulse enables a detectable signal (i.e., a "no-click" event). In this gedankenexperiment, the "no-click" event heralds the presence of a single photon in the output port of the beamsplitter.

This simple example highlights the role of dark counts and detector inefficiency when heralding on zero. A dark count at $D_{1}$ alongside a signal from $D_{r e f}$ will register as a "click," leading us to discard the output even if the single photon was transmitted. This reduces the probability of success for our device. Detector inefficiency, meanwhile, will cause us to miss reflected photons and erroneously herald none in the output. This reduces the fidelity of states heralded by a "no-click" event. Significantly, the effects of dark counts and inefficiency are reversed from conventional heralding on single photons [26, 27, so what makes $D_{1}$ a good "zero photon detector" may be defined by different criteria. For example, techniques which reduce losses at the cost of increased background noise may be beneficial in certain applications [13].

To quantify the effects of dark counts and detector inefficiency, we can measure the output state with an auxiliary detector $D_{2}$ and analyze the statistics of many trials, as shown in Figure $2 \mathrm{~b}$. First, detection events from $D_{r e f}, D_{1}$ and $D_{2}$ are processed by time-to-digital converters (TDCs) and stored as time tags relative to a master clock. This timing information allows us to reconstruct a sequence of "click" and "no-click" events alongside every output measurement [24]. The first table in Figure $2 \mathrm{~b}$ shows such a record with various $D_{1}$ detection errors marked with asterisks. The consequences of these errors appear in the subset of data selected based on a "no-click" event at $D_{1}$, shown in the second table. The number of rows in this second table reflects the prob- ability of success, and its output column should ideally contain only single photons. Dark counts at $D_{1}$ (e.g., Pulse \#5) are simply excluded from the subset of successful trials, reducing its size. Meanwhile, undetected photons at $D_{1}$ due to detector inefficiency (e.g., Pulses \#1 and \#9) are accepted as "successful" trials despite no photons being transmitted, corrupting the heralded output state. A more quantitative analysis of the effects of dark counts and detector inefficiency is shown in Appendix $\mathrm{A}$.

In a realistic experiment with conventional siliconbased avalanche photodiodes (i.e., SPCMs), the $D_{1}$ dark count rates $\left(\sim 10^{2} \mathrm{~Hz}\right)$ are much smaller than typical trial rates (often $10^{4}-10^{7} \mathrm{~Hz}$ ). Thus, the change in probability of success will be negligible for many applications. In contrast, $D_{1}$ efficiency (typically $\sim 50 \%$ ) impacts a significant fraction of the trials and will drastically reduce the output fidelity in a realistic "heralding on zero" process. Consequently, the remainder of this paper will focus on the effects of $D_{1}$ inefficiency on the heralded output.

\section{EXPERIMENTAL DEMONSTRATION}

In order to experimentally demonstrate the role of limited detector efficiency in heralding on zero, we use a variant of the well-known Hong-Ou-Mandel (HOM) interferometer 25. As pictured in Figure 3, two photons are mixed at a 50/50 beamsplitter, resulting in four possible two-photon output amplitudes which we denote $R R$, $T T, R T$, and $T R$, with $R$ and $T$ implying single photon reflection and transmission, as usual [25. The photons are assumed to be indistinguishable, except for the temporal degree of freedom which is controlled by a relative delay $\Delta t$. When $\Delta t=0$, the beamsplitter statistics correspond to those of indistinguishable bosons, resulting 


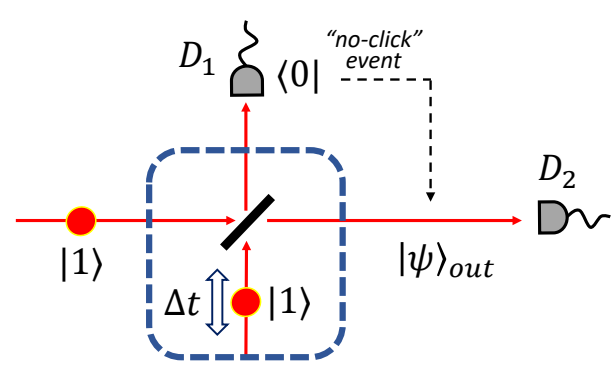

FIG. 3. Conceptual illustration of heralding on zero in a modified Hong-Ou-Mandel (HOM) interferometer. Two identical photons (red circles) enter a 50/50 beamsplitter, and are made temporally distinguishable by a tunable delay $\Delta t$ in the lower input mode. As the delay is scanned through $\Delta t=0$, the probability of heralding on zero doubles, resulting in a dramatic "peak" in the heralded $D_{2}$ event rate. However, the relative height of the peak rapidly degrades as a function of $D_{1}$ efficiency, providing a method for quantifying the role of detector efficiency in the heralding on zero process.

in the suppression of the $R R$ and $T T$ amplitudes (i.e., bosonic "bunching" [28]). Experimentally, this leads to the well-known HOM "dip" in coincident detections between $D_{1}$ and $D_{2}$ as $\Delta t$ is scanned through zero [25].

Instead of measuring coincidence counts while scanning $\Delta t$, here we herald on zero photons in $D_{1}$, and then study the single count rates in $D_{2}$ (see Figure 3). In the idealized case, heralding on zero in $D_{1}$ simply means there are two photons in the output mode (i.e., headed to $D_{2}$ ) [17, 29]. What is interesting, however, is that the probability of this occurring depends on the value of $\Delta t$ : when $\Delta t$ is large, heralding on zero in $D_{1}$ only occurs for one of the 4 possible two-photon amplitudes; when $\Delta t=0$, it occurs for one of only 2 possible two-photon amplitudes. Consequently, in an experiment with many repeated trials, the measurement process illustrated in Figure 3 should show a dramatic "peak" (i.e., doubling) in the heralded $D_{2}$ click rate as $\Delta t$ is scanned through zero time delay.

For our purposes, the key point is that the quality of this peak critically depends on the ability to effectively herald on zero, and the idealized doubling in counting rates rapidly degrades with $D_{1}$ efficiency, $\eta_{1}$. Consequently, studying the relative peak height as a function of $\eta_{1}$ provides a robust metric for demonstrating the role of detector efficiency in heralding on zero.

This measurement can be accomplished with the same techniques described in Section [II but with several additional considerations. First, we must account for the probability of "no-click" events given more than one photon at a non-PNR single-photon detector. This is given by $p(N C \mid n)=(1-\eta)^{n}$ for $n$ photons and negligible dark count probability [30, 31. Here, the effective detector efficiency $\eta$ includes quantum efficiency, spatial mode overlap, and other forms of photon loss in the detection channel 32. Next, our output measurement is no longer perfect, so the probability $p(N C \mid n)$ must be applied to both the heralding detector $D_{1}$ and the output detector $D_{2}$ (which is also inefficient and non-PNR). Lastly, our input state signaled by detector $D_{\text {ref }}$ (see Fig. 2) now corresponds to photon pairs derived from a probabilistic PDC source. We approximate the output of this source as a superposition of the vacuum and a single photon pair, ignoring higher-order terms [33]:

$$
|\psi\rangle_{i n} \approx \sqrt{1-\gamma}|00\rangle+\sqrt{\gamma}|11\rangle
$$

where $\gamma$ is the pair emission probability per pulse ( $\sim 10^{-4}$ in our experiment). The input state is further modified by finite coupling efficiencies $\kappa_{1}$ and $\kappa_{2}$, limited by the broadband pulsed PDC process [34, 35], and separate from detector efficiencies $\eta_{1}$ and $\eta_{2}$ at the outputs.

Incorporating all of this into our analysis (see Appendix $B$, we obtain the probability of a $D_{2}$ click conditioned on a "no-click" at $D_{1}$ :

$$
P\left(C_{2} \mid N C_{1}\right) \approx \frac{\gamma \eta_{2}^{\prime}}{4}\left[4-\eta_{2}^{\prime}-2 \eta_{1}^{\prime}+\nu\left(2 \eta_{1}^{\prime}-\eta_{2}^{\prime}\right)\right],
$$

where $\eta_{i}^{\prime} \equiv \sqrt{\kappa_{1} \kappa_{2}} \eta_{i}$, and $\nu$ is a function of the time delay that captures the degree of indistinguishability of the two input photons. This parameter ranges from $\nu=1$ at $\Delta t=0$ to $\nu=0$ when $\Delta t$ is large. (For reference, the conventional HOM "dip" using the same apparatus would have the form $(1-\nu)$ as $\Delta t$ is scanned across zero.) The above expression also contains two approximations that are both valid in our experiments: first, that input coupling is roughly balanced $\left(\kappa_{1} \approx \kappa_{2}\right)$, and second, that $\gamma \ll 1$.

From Equation 2 it can be seen that when $\eta_{1}^{\prime}=1$ (perfect efficiency), we find that $P\left(C_{2} \mid N C_{1}\right) \propto(1+\nu)$, indicating the high-visibility peak (doubling) in counts that does not depend on $\eta_{2}^{\prime}$. However, as $\eta_{1}^{\prime}$ decreases, the relative peak height also decreases monotonically until $\eta_{1}^{\prime}=0$. At this point, the heralding process is completely ineffective and we are simply observing the ordinary singles counting rate of $D_{2}$. This change of peak height as a function of $\eta_{1}^{\prime}$ is what we observe in our experiment.

The full experimental setup is shown in Figure 4. Our input state consists of down-converted photon pairs with center wavelength $780 \mathrm{~nm}$, generated via degenerate Type-I PDC using a BBO crystal. The crystal is pumped with a $100 \mathrm{MHz}$ train of UV pulses $(\sim 150 \mathrm{fs}$ duration, $390 \mathrm{~nm}$ center wavelength), derived from a frequency-doubled mode-locked fiber laser (Menlo Systems C-Fiber 780). The pairs are collected and focused into two single-mode fibers, with associated coupling efficiencies $\kappa_{1}$ and $\kappa_{2}$. The HOM interferometer consists of a 50-50 fiber coupler, as well as a pre-fiber time delay $\Delta t$ implemented with two translating glass wedges. After the HOM interferometer lie two detection channels; each consists of a free-space U-bench with 25-nm-bandwidth rectangular bandpass filters centered near $780 \mathrm{~nm}$, before finally being coupled into multimode fibers and directed to SPCMs $D_{1}$ and $D_{2}$ (silicon avalanche photodiodes, Excelitas SPCM-AQ4C). Detection signals, including the $100 \mathrm{MHz}$ mode-locking reference signal from 


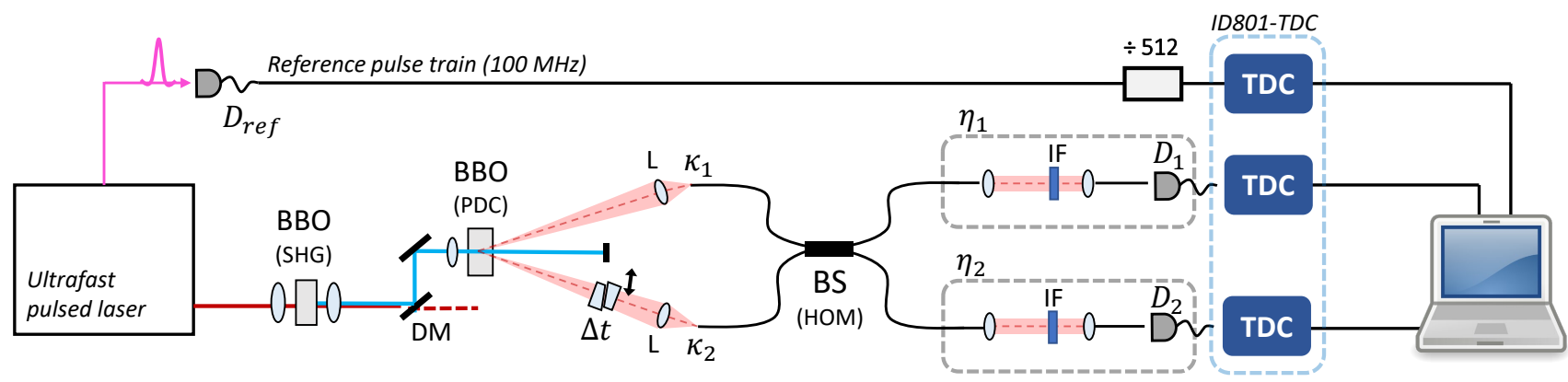

FIG. 4. Experimental implementation of the modified HOM interferometer using photon pairs from a pulsed Parametric DownConversion (PDC) source, and a single-mode 3dB fiber coupler as the 50/50 beamsplitter (BS). The output of an ultrafast pulsed laser (100 MHz, $780 \mathrm{~nm}$ ) undergoes Second Harmonic Generation (SHG) in order to produce a train of PDC pump pulses at $390 \mathrm{~nm}$. The PDC crystal (BBO, Type I phase matching) produces photon pairs at $780 \mathrm{~nm}$. Detectors $D_{1}, D_{2}$, and $D_{r e f}$, as well as the TDCs are used to implement the measurement and data post-processing techniques illustrated in Figure 2 2 . DMdichroic mirror used to isolate UV pump pulses; L- various lenses; $\Delta t$ - translating glass wedges; IF- narrowband interference filters centered near $780 \mathrm{~nm}$; “ $\div 512 "$ - data frequency divider.

$D_{\text {ref }}$, are recorded with TDCs with 81 ps timebin resolution (IDQuantique, model ID801). Since $100 \mathrm{MHz}$ exceeds the data bandwidth of our TDCs, the reference signal is buffered by frequency divider (Valon 3010a), so we tag one of every 512 pulses and reconstruct the pulse train in data processing.

The use of time tags and the data processing techniques of Fig. $2 \mathrm{~b}$ offer several practical advantages. For example, by using an external reference signal, "no-click" events can be identified for the heralding detector, and the relevant output measurement statistics can be seen in post-selection 24]. In addition, we can arbitrarily extend detector dead time in post-processing, mitigating the effects of afterpulsing [36]. In our experiment, we ignore 5 pulses following a click in either detector to account for SPCM detector dead times of $\sim 50$ ns. Furthermore, the reference pulse train (mode-locking signal) can be used as a virtual gating signal for each free-running detector. We only accept counts within a $2 \mathrm{~ns}$ window after each reference pulse, discarding outlying dark counts. In our system, virtual gating reduces dark counts rates in each detector to a negligible rate of $\sim 60$ per second, or $<10^{-6}$ per pulse. As a final note, these technical advantages are offset to some extent by significant storage requirements for reference pulse time tags, which can be mitigated with various techniques 37 .

\section{RESULTS AND ANALYSIS}

We first align and optimize our apparatus by performing conventional Hong-Ou-Mandel measurements of the coincidence counting rate between $D_{1}$ and $D_{2}$ as a function of $\Delta t$. The optimized results are shown in Figure 5 , where each data point is displayed as an average counting rate, calculated from 20 seconds of stored time tags and a coincidence window of 2 ns. The results show a high-

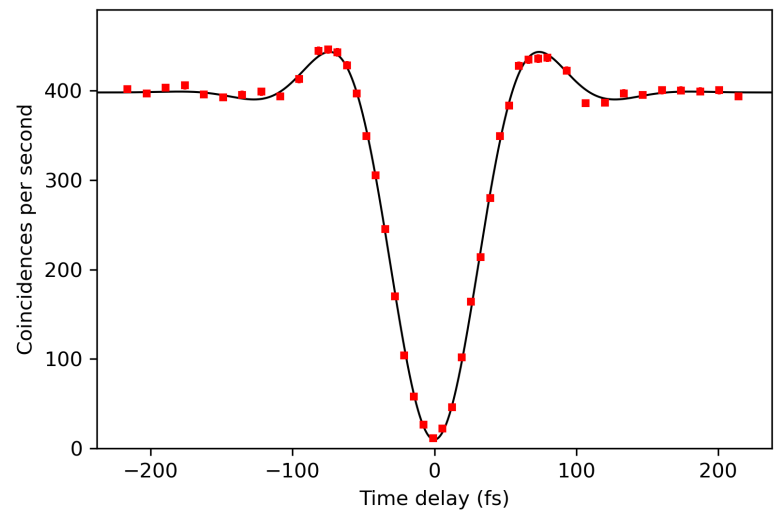

FIG. 5. Alignment and testing of the setup using measurements of the conventional Hong-Ou-Mandel "dip" in coincidence counts between detectors $D_{1}$ and $D_{2}$. Experimental data points are in red. The solid black line is a least-squares fit using a simple model that takes into account the nonGaussian transmission profiles of the narrowband interference filters. The model gives a HOM dip visibility of $(97.5 \pm 0.6) \%$, indicating a high degree of indistinguishability.

quality HOM "dip" with a visibility of $(97.5 \pm 0.6) \%$. This provides a measure of the indistinguishability of the photons and, importantly, an experimental upper bound for $\nu$ of $\nu_{\max }=0.975$.

The main results of our "heralding on zero" study are summarized in Figure 6. The two experimental curves (blue and red data points) are derived from the same recorded time tags used to produce the results of Figure 5. For convenience, we convert the probability per pulse, $P\left(C_{2} \mid N C_{1}\right)$ of Eqn 2, into heralded $D_{2}$ clicks per second using the repetition rate of the experiment. The data shows how the size of the expected "peak" in the heralded $D_{2}$ click rate is reduced with decreasing $D_{1}$ efficiency $\eta_{1}^{\prime}$. 


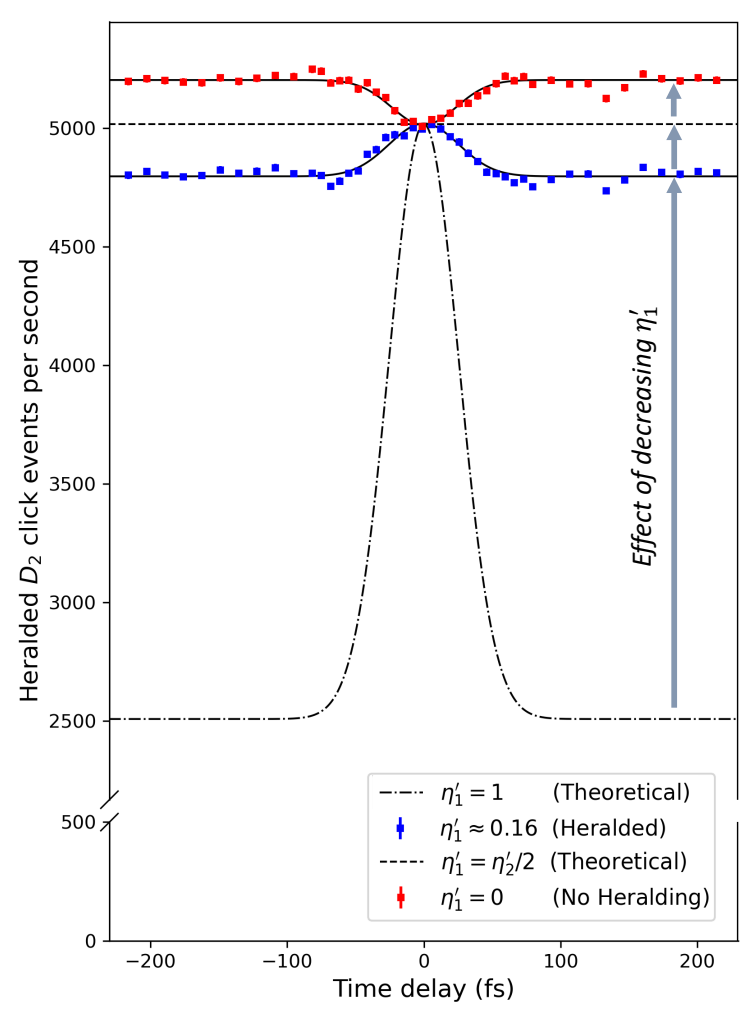

FIG. 6. Demonstration of the deleterious effects of detector inefficiency when heralding on zero in the HOM interferometer. The 4 curves show the measured (or predicted) click rates at detector $D_{2}$ for different values of heralding detector efficiency $\eta_{1}^{\prime}$. For perfect efficiency $\eta_{1}^{\prime}=1$ (lowest curve), the heralded click rate doubles when $\Delta t=0$, resulting in a dramatic "peak" in the predicted curve. As $\eta_{1}^{\prime}$ is decreased, this effective peak height is reduced. The blue (lower) data points are experimental measurements taken with $\eta_{1}^{\prime} \approx 0.16$. As $\eta_{1}^{\prime}$ is further reduced to the value $\eta_{1}^{\prime}=\eta_{2}^{\prime} / 2$, the peak flattens (dashed line), and then transitions to a "dip" when $\eta_{1}^{\prime}<\eta_{2}^{\prime} / 2$. The red (upper) data points correspond to the limiting case $\eta_{1}^{\prime}=0$ (i.e., no heralding). In all cases, output detector efficiency is fixed at the experimentally determined value $\eta_{2}^{\prime} \approx 0.15$.

For reference, the idealized case of $\eta_{1}^{\prime}=1$ is shown by the dashed theory curve in the lower part of Figure 6. This is simply the prediction given by Equation 2 with $\gamma$ and $\eta_{2}^{\prime}$ experimentally determined. When $\Delta t=0$, the peak of the dashed theory curve shows the ideal doubling in click rate.

The blue data points are our experimentally measured values of $P\left(C_{2} \mid N C_{1}\right)$ with a low value of $\eta_{1}^{\prime}$. The idealized doubling in peak height is significantly reduced by raising the count rates at large time delays (the "wings") relative to the count rates at the central point when $\Delta t=0$. We quantify this reduction by taking the ratio of Eqn. 2 at zero and large time delays (Appendix B], which we call the center-to-wings ratio $\xi$ :

$$
\xi \approx 1+\nu_{\max }\left(\frac{2 \eta_{1}^{\prime}-\eta_{2}^{\prime}}{4-2 \eta_{1}^{\prime}-\eta_{2}^{\prime}}\right)
$$

In the ideal case $\eta_{1}^{\prime}=1$, the above simplifies to $1+\nu_{\max }$, but decreases monotonically with heralding detector efficiency. As $\eta_{1}^{\prime}$ worsens and count rates on the wings continue to rise, eventually one actually expects a relative "dip" in counts for zero time delay, $\xi<1$. This occurs in the worst case $\eta_{1}^{\prime}=0$, where heralding on zero is completely ineffective and admits all possible states into the output. This limiting case of no heralding is shown by the red data points in Figure 6, and corresponds to the dip in ordinary singles counting rates first observed by Resch et al. 30 and Kim et al. 38.

For simplicity, we use basic Gaussian fits to our experimental data and find $\xi$ values of approximately 1.05 and 0.96 for our peak (blue) and dip (red), which correspond to effective detector efficiencies $\eta_{1}^{\prime} \approx 0.16$ and $\eta_{2}^{\prime} \approx 0.15$. This is consistent with the nominal detection efficiencies of our silicon avalanche photodiodes $(\sim 50 \%$ at $780 \mathrm{~nm}$ ), combined with limited coupling efficiencies $\left(\kappa_{1}\right.$ and $\left.\kappa_{2} \sim 50 \%\right)$ [34, 35], and U-bench transmission $(\sim 65 \%)$. The theoretical curves in Figure 6 are calculated using the same value of $\eta_{2}^{\prime}$ while varying heralding detector efficiency [39].

In Figure 6, both the "peak" (blue) and "dip" (red) results appear to share the same value at zero time delay. This is due to the fact that our model for heralded statistics in Eqn. 2 is equivalent to the coincidence counting rate subtracted from the ordinary singles counting rates (see Appendix B] [40. Thus the difference between red and blue data points is nearly zero at zero time delay, and matches the experimental coincidence counting rate of Figure 5. Additionally, since $\xi$ evolves continuously with heralding efficiency, there is a value of $\eta_{1}^{\prime}$ at which the interference pattern transitions from a "peak" to a "dip." This condition is shown by the flat dashed line in Figure 6. By inspection of Eqn. 3, this occurs when $\eta_{1}^{\prime}=\eta_{2}^{\prime} / 2$. At this point, a heralded $D_{2}$ "click" event is equally likely to result from "bunched" ( $T R$ or $R T$ ) or "antibunched" (TT or RR) two-photon amplitudes, and so interference no longer appears in this measurement.

This transition is further theoretically explored in Figure 7. where $\xi$ is plotted as a function of the ratio $\eta_{1}^{\prime} / \eta_{2}^{\prime}$ for a wide range of efficiencies extending beyond our experimental capabilities. For simplicity, here we also assume $\nu_{\max }=1$. Three curves are shown for different values of output efficiency $\eta_{2}^{\prime}$, and all of them pass through the non-interference condition point $\xi=1$ when $\eta_{1}^{\prime}=\eta_{2}^{\prime} / 2$. Above this threshold, we observe a peak in heralded $D_{2}$ click events. The upper bound of $\xi=2$ is achieved for perfect heralding efficiency $\eta_{1}^{\prime}=1$. Below the threshold, the efficiency of the output detector becomes most important as heralding efficiency drops to zero. Although the average photon flux at detector $D_{2}$ remains constant with no heralding $\left(\eta_{1}^{\prime}=0\right)$, the lack of PNR results in a dip in singles rates. For the ex- 


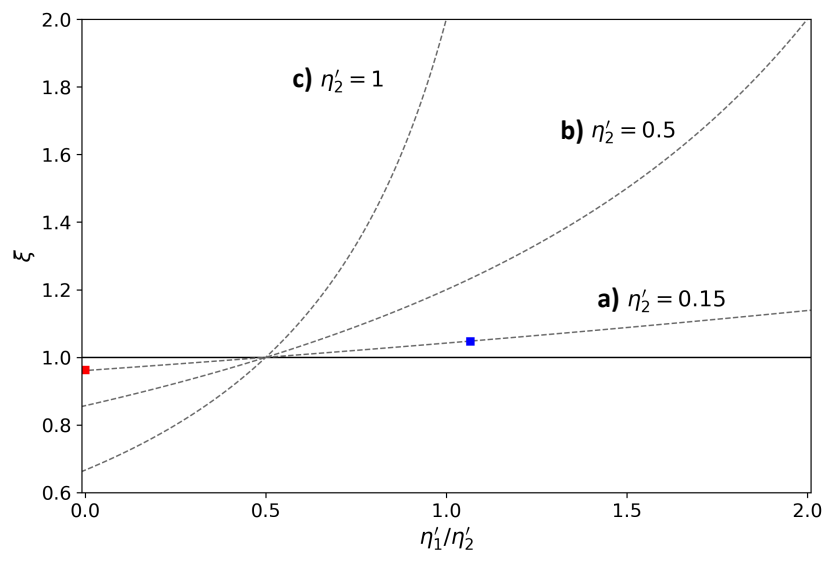

FIG. 7. Theoretical plots of center-to-wings ratio $\xi$ as a function of $\eta_{1}^{\prime} / \eta_{2}^{\prime}$, shown for three values of output detector efficiency: a) the experimental value $\eta_{2}^{\prime}=0.15$, b) an intermediate efficiency $\eta_{2}^{\prime}=0.5$ and c) the ideal case $\eta_{2}^{\prime}=1$. Interestingly, no interference can be observed (i.e., $\xi=1$ ) when $\eta_{1}^{\prime} / \eta_{2}^{\prime}=1 / 2$, regardless of the actual detector efficiency values. When $\eta_{1}^{\prime} / \eta_{2}^{\prime}>1 / 2$, dramatic "peaks" $(\xi>1)$ can be observed in the $D_{2}$ click rate with a maximum of $\xi=2$ for the ideal case of $\eta_{1}^{\prime}=1$ and $\nu_{\max }=1$. When $\eta_{1}^{\prime} / \eta_{2}^{\prime}<1 / 2$, the "peak" transitions to a "dip" $(\xi<1)$, with a minimum value of $\xi=2 / 3$ when $\eta_{2}^{\prime}=1$ and $\eta_{1}^{\prime}=0$ (i.e., equivalent to no heralding). For reference, the two points (red and blue) on curve (a) correspond to observed data in Figure 6.

treme case $\left(\eta_{1}^{\prime}, \eta_{2}^{\prime}\right)=(0,1)$, we see the largest possible dip of $\xi=2 / 3$. This lower bound can be explained with the two-photon amplitude model: for large $\Delta t, 3$ of 4 possibilities send at least one photon to detector $D_{2}$; for $\Delta t=0$, this reduces to 1 of 2 possibilities, yielding the above ratio. This dramatic $D_{2}$ singles rate "dip" is present in all conventional HOM experiments with SPCMs, but is significantly reduced by limited $D_{2}$ efficiency and other losses [30, 38, 41].

\section{CONCLUSIONS}

While the concept of heralding on the detection of zero photons is fairly straightforward, the implementation with current technology presents a number of challenges. We have investigated a basic approach that combines the detection of a reference (timing) signal with the simultaneous absence of a detection in a commercial SPCM (silicon-based avalanche photodiodes). This combination successfully registers a "no-click" event that can then be used to actively herald the desired output state in various applications.

In this approach, the role of dark counts in the heralding detector merely reduces the probability of success [13. In contrast, the role of detector inefficiency severely reduces the fidelity of the heralded output states. In some sense, this makes experimental heralding on zero much more difficult than conventional heralding on one photon, where these roles are reversed.
We experimentally quantified the effects of detector inefficiency in heralding on zero using a variation of the HOM interferometer as a test system. The results showed a rapid deterioration of the relevant quantum interference effects as the heralding detector efficiency is reduced. Extensions of this demonstration to practical systems in quantum communications [12, 13] and quantum state generation [42, 43] support the need for current research efforts in increasing detector efficiency [44, 45]. This is particularly relevant for more complex scenarios, such as LOQC applications that may require simultaneous heralding on zero in multiple heralding channels.

\section{ACKNOWLEDGMENTS}

This work was supported by the National Science Foundation under grant number PHY-2013464.

\section{Appendix A: Heralding on Zero Statistics}

Here we present a quantitative analysis to justify the conclusions of Section IIIregarding detector efficiency and dark counts. We go back to the more general case of Figure 1 1 , with arbitrary input states. We start with the probability that a non-PNR detector $D_{1}$ will not click given exactly $n$ photons enter the detection channel 30 , 31:

$$
p(N C \mid n)=(1-d)(1-\eta)^{n},
$$

where $d$ is the probability of a dark count, assumed to be independent of $n$, and $\eta$ is the heralding detector efficiency. The probability of success $P_{s}$ is simply the total likelihood of a no-click event, $P(N C)$. Assuming a probability $P_{n}$ of $n$ photons appearing in the heralding mode:

$$
P_{s}=\sum_{n=0}^{\infty}(1-d)(1-\eta)^{n} P_{n}
$$

Thus, the probability of success decreases linearly with dark counts. However, only detector efficiency affects the quality (i.e., fidelity) of the heralded output. From Bayes' rule, we can calculate the probability of heralding the desired state $|\psi\rangle_{\text {out }}$ given a no-click event:

$$
\begin{aligned}
P\left(\psi_{\text {out }} \mid N C\right) & =\frac{P\left(N C \mid \psi_{\text {out }}\right) \cdot P\left(\psi_{\text {out }}\right)}{P(N C)}=\frac{(1-d) \cdot P_{0}}{P_{s}} \\
& =\frac{P_{0}}{\sum_{n=0}^{\infty}(1-\eta)^{n} P_{n}}
\end{aligned}
$$

With unit detector efficiency $\eta=1$, we see that Eqn. A3 is also unity, meaning a no-click event always heralds the desired output state. As $\eta$ decreases, however, this probability decreases as no-click events herald mixed states with added noise.

For example, in the single-photon gedankenexperiment of Figure $2 \mathrm{a}$, using a 50/50 beamsplitter, we have $P_{0}=$ 
$P_{1}=1 / 2$. Thus, Eqn. A2 gives $P_{s}=(1-d)\left(2-\eta_{1}\right) / 2$ and Eqn. A3 gives $P\left(\psi_{\text {out }} \mid N C_{1}\right)=1 /\left(2-\eta_{1}\right)$.

In this idealized setup, the degraded output state can be adequately characterized with the counting rates of one single-photon detector, $D_{2}$, accounting for additional losses. For a realistic single-photon source, which may also include small, undesired zero-photon and twophoton contributions, this would be better accomplished by a $g^{(2)}$ measurement in the output mode [27].

\section{Appendix B: HOM Heralded Click-rate Calculations}

Including the coupling efficiencies $\kappa_{1}$ and $\kappa_{2}$, the probabilities $P(m, n)$ of finding $m$ and $n$ photons in the two HOM interferometer outputs are given by:

$$
\begin{gathered}
P(1,1)=\frac{\gamma \kappa_{1} \kappa_{2}}{2}(1-\nu) \\
P(1,0)=P(0,1)=\frac{\gamma}{2}\left[\kappa_{1}\left(1-\kappa_{2}\right)+\left(1-\kappa_{1}\right) \kappa_{2}\right] \\
P(2,0)=P(0,2)=\frac{\gamma \kappa_{1} \kappa_{2}}{4}(1+\nu)
\end{gathered}
$$

By applying Eqn. A1 and neglecting dark counts, we can obtain the probability of a click at either detector (singles), and a joint click event (coincidence):

$$
\begin{array}{r}
P\left(C_{i}\right)=\frac{\gamma \eta_{i} \tilde{\kappa}}{4}\left[\frac{4 \bar{\kappa}}{\tilde{\kappa}}-(1+\nu) \eta_{i} \tilde{\kappa}\right] \\
P\left(C_{1} \wedge C_{2}\right)=\frac{\gamma \eta_{1} \eta_{2} \tilde{\kappa}^{2}}{2}(1-\nu)
\end{array}
$$

where the input coupling efficiencies have been combined into $\bar{\kappa}$ and $\tilde{\kappa}$, the arithmetic and geometric mean, respectively. As long as the coupling efficiencies are roughly equal, we can approximate that $\bar{\kappa}=\tilde{\kappa}$.

Next, we find the probability of a $D_{2}$ click conditioned on a $D_{1}$ no-click in terms of Eqns. B1 and B2, once again using Bayes' rule:

$$
P\left(C_{2} \mid N C_{1}\right)=\frac{P\left(C_{2}\right)-P\left(C_{1} \wedge C_{2}\right)}{1-P\left(C_{1}\right)}
$$

With small pair probability $\gamma \ll 1$, the denominator (i.e., the probability of success) can be taken as unity. This last approximation gives us Eqn. 2, which is equal to $P\left(C_{2}\right)-P\left(C_{1} \wedge C_{2}\right)$; this is simply the coincidence counting rate subtracted from the $D_{2}$ singles counting rate.

The center-to-wings ratio $\xi$ of Eqn. 3 is defined:

$$
\xi \equiv \frac{\left.P\left(C_{2} \mid N C_{1}\right)\right|_{\nu=\nu_{\max }}}{\left.P\left(C_{2} \mid N C_{1}\right)\right|_{\nu=0}} \approx 1+\nu_{\max }\left(\frac{2 \frac{\eta_{1}^{\prime}}{\eta_{2}^{\prime}}-1}{\frac{4}{\eta_{2}^{\prime}}-2 \frac{\eta_{1}^{\prime}}{\eta_{2}^{\prime}}-1}\right)
$$

where the above form illustrates the dependence on efficiency mismatch $\eta_{1}^{\prime} / \eta_{2}^{\prime}$, illustrated in Fig. 7 .

Note that the approximation in Eqn. B4 only holds for $\gamma \ll 1$ and $\kappa_{1} \approx \kappa_{2}$. When these conditions are not met, the presence of higher-order terms (multiple pairs) in the PDC process and largely asymmetric coupling losses can affect both $\xi$ and the conventional HOM dip visibility in these types of experiments 46].
[1] P. Kok and B. W. Lovett, Introduction to Optical Quantum Information Processing (Cambridge University Press, 2010).

[2] M. Dakna, L. Knöll, and D.-G. Welsch, Quantum state engineering using conditional measurement on a beam splitter, The European Physical Journal D - Atomic, Molecular, Optical and Plasma Physics 3, 295 (1998)

[3] C. K. Hong and L. Mandel, Experimental realization of a localized one-photon state, Phys. Rev. Lett. 56, 58 (1986)

[4] T. B. Pittman, B. C. Jacobs, and J. D. Franson, Single photons on pseudodemand from stored parametric downconversion, Phys. Rev. A 66, 042303 (2002)

[5] A. L. Migdall, D. Branning, and S. Castelletto, Tailoring single-photon and multiphoton probabilities of a singlephoton on-demand source, Phys. Rev. A 66, 053805 (2002)

[6] A. Ourjoumtsev, R. Tualle-Brouri, J. Laurat, and P. Grangier, Generating optical schrödinger kittens for quantum information processing, Science 312, 83 (2006), https://science.sciencemag.org/content/312/5770/83.full.pd

[7] D. T. Pegg, L. S. Phillips, and S. M. Barnett, Optical state truncation by projection synthesis, Phys. Rev. Lett. 81, 1604 (1998)

[8] E. Knill, R. Laflamme, and G. J. Milburn, A scheme for efficient quantum computation with linear optics, Nature
409, 46 (2001)

[9] S. M. Young, M. Sarovar, and F. Léonard, Design of high-performance photon-number-resolving photodetectors based on coherently interacting nanoscale elements, ACS Photonics 7, 821 (2020)

[10] G. S. Thekkadath, M. E. Mycroft, B. A. Bell, C. G. Wade, A. Eckstein, D. S. Phillips, R. B. Patel, A. Buraczewski, A. E. Lita, T. Gerrits, S. W. Nam, M. Stobińska, A. I. Lvovsky, and I. A. Walmsley, Quantum-enhanced interferometry with large heralded photon-number states, $\mathrm{npj}$ Quantum Information 6, 89 (2020),

[11] F. Mattioli, Z. Zhou, A. Gaggero, R. Gaudio, S. Jahanmirinejad, D. Sahin, F. Marsili, R. Leoni, and A. Fiore, Photon-number-resolving superconducting nanowire detectors, Superconductor Science and Technology 28, 104001 (2015)

[12] M. Mičuda, I. Straka, M. Miková, M. Dušek, N. J. Cerf, J. Fiurášek, and M. Ježek, Noiseless loss suppression in quantum optical communication, Phys. Rev. Lett. 109, 180503 (2012)

df [13] C. N. Gagatsos, J. Fiurášek, A. Zavatta, M. Bellini, and N. J. Cerf, Heralded noiseless amplification and attenuation of non-gaussian states of light, Phys. Rev. A 89, $062311(2014)$

[14] R. A. Brewster, I. C. Nodurft, T. B. Pittman, and J. D. Franson, Noiseless attenuation using an optical paramet- 
ric amplifier, Phys. Rev. A 96, 042307 (2017)

[15] A. E. Lita, A. J. Miller, and S. W. Nam, Counting nearinfrared single-photons with $95 \%$ efficiency, Opt. Express 16, 3032 (2008).

[16] M. Lamperti, A. Allevi, M. Bondani, R. Machulka, V. Michálek, O. Haderka, and J. Peřina, Optimal subpoissonian light generation from twin beams by photonnumber resolving detectors, J. Opt. Soc. Am. B 31, 20 (2014)

[17] M. von Helversen, J. Böhm, M. Schmidt, M. Gschrey, J.-H. Schulze, A. Strittmatter, S. Rodt, J. Beyer, T. Heindel, and S. Reitzenstein, Quantum metrology of solid-state single-photon sources using photon-numberresolving detectors, New Journal of Physics 21, 035007 (2019)

[18] A. Allevi, A. Andreoni, M. Bondani, M. G. Genoni, and S. Olivares, Reliable source of conditional states from single-mode pulsed thermal fields by multiple-photon subtraction, Phys. Rev. A 82, 013816 (2010).

[19] Y. Zhai, F. E. Becerra, B. L. Glebov, J. Wen, A. E. Lita, B. Calkins, T. Gerrits, J. Fan, S. W. Nam, and A. Migdall, Photon-number-resolved detection of photonsubtracted thermal light, Opt. Lett. 38, 2171 (2013).

[20] Y. I. Bogdanov, K. G. Katamadze, G. V. Avosopiants, L. V. Belinsky, N. A. Bogdanova, A. A. Kalinkin, and S. P. Kulik, Multiphoton subtracted thermal states: Description, preparation, and reconstruction, Phys. Rev. A 96, 063803 (2017).

[21] J. Hloušek, M. Ježek, and R. Filip, Work and information from thermal states after subtraction of energy quanta, Scientific Reports 7, 13046 (2017)

[22] O. S. Magaña-Loaiza, R. d. J. León-Montiel, A. PerezLeija, A. B. U'Ren, C. You, K. Busch, A. E. Lita, S. W. Nam, R. P. Mirin, and T. Gerrits, Multiphoton quantumstate engineering using conditional measurements, npj Quantum Information 5, 80 (2019).

[23] K. G. Katamadze, G. V. Avosopiants, N. A. Bogdanova, Y. I. Bogdanov, and S. P. Kulik, Multimode thermal states with multiphoton subtraction: Study of the photon-number distribution in the selected subsystem, Phys. Rev. A 101, 013811 (2020).

[24] J. Sinclair, D. Angulo, K. Thompson, K. Bonsma-Fisher, A. Brodutch, and A. M. Steinberg, Measuring the time atoms spend in the excited state due to a photon they don't absorb (2020), arXiv:2010.02200 [quant-ph].

[25] C. K. Hong, Z. Y. Ou, and L. Mandel, Measurement of subpicosecond time intervals between two photons by interference, Phys. Rev. Lett. 59, 2044 (1987)

[26] V. D'Auria, O. Morin, C. Fabre, and J. Laurat, Effect of the heralding detector properties on the conditional generation of single-photon states, The European Physical Journal D 66, 249 (2012).

[27] A. M. Brańczyk, T. C. Ralph, W. Helwig, and C. Silberhorn, Optimized generation of heralded fock states using parametric down-conversion, New Journal of Physics 12, 063001 (2010)

[28] R. Loudon, Fermion and boson beam-splitter statistics, Phys. Rev. A 58, 4904 (1998)

[29] G. Di Giuseppe, M. Atatüre, M. D. Shaw, A. V. Sergienko, B. E. A. Saleh, M. C. Teich, A. J. Miller, S. W. Nam, and J. Martinis, Direct observation of photon pairs at a single output port of a beam-splitter interferometer, Phys. Rev. A 68, 063817 (2003)

[30] K. J. Resch, J. S. Lundeen, and A. M. Steinberg, Ex- perimental observation of nonclassical effects on singlephoton detection rates, Phys. Rev. A 63, 020102(R) (2001)

[31] M. J. Stevens, Chapter 2 - photon statistics, measurements, and measurements tools, in Single-Photon Generation and Detection, Experimental Methods in the Physical Sciences, Vol. 45, edited by A. Migdall, S. V. Polyakov, J. Fan, and J. C. Bienfang (Academic Press, 2013) pp. 25-68.

[32] M. Ware and A. Migdall, Single-photon detector characterization using correlated photons: the march from feasibility to metrology, Journal of Modern Optics 51, 1549 (2004).

[33] C. C. Gerry and P. L. Knight, Introductory quantum optics (Cambridge University Press, Cambridge, 2004).

[34] T. B. Pittman, B. C. Jacobs, and J. D. Franson, Heralding single photons from pulsed parametric downconversion, Optics Communications 246, 545 (2005)

[35] F. A. Bovino, P. Varisco, A. Maria Colla, G. Castagnoli, G. Di Giuseppe, and A. V. Sergienko, Effective fibercoupling of entangled photons for quantum communication, Optics Communications 227, 343 (2003)

[36] G. Ulu, A. V. Sergienko, and M. S. Ünlü, Influence of hot-carrier luminescence from avalanche photodiodes on time-correlated photon detection, Opt. Lett. 25, 758 (2000)

[37] M. Wahl, T. Röhlicke, S. Kulisch, S. Rohilla, B. Krämer, and A. C. Hocke, Photon arrival time tagging with many channels, sub-nanosecond deadtime, very high throughput, and fiber optic remote synchronization, Review of Scientific Instruments 91, 013108 (2020), https://doi.org/10.1063/1.5121412.

[38] Y.-H. Kim and W. P. Grice, Observation of correlatedphoton statistics using a single detector, Phys. Rev. A 67, 065802 (2003).

[39] In practice, our ability to fix one effective detector efficiency while varying the other is limited, since they both depend on $\kappa_{1}$ and $\kappa_{2}$. However, it is more instructive to consider the efficiencies as independent, as if all losses occur in the detection channels and not before the HOM interferometer. Our analysis applies in either case.

[40] Another explanation for this fixed point is more fundamental: including all higher-order terms from the PDC process, at $\Delta t=0$ the outputs of the beamsplitter should ideally be disentangled and thus heralding on $D_{1}$ should not affect the state in the other output mode [47. It is only by comparison to nonzero time delays that we gain information about the heralding capability of detector $D_{1}$.

[41] P. S. K. Lee and M. P. van Exter, Spatial labeling in a two-photon interferometer, Phys. Rev. A 73, 063827 (2006)

[42] W. Ye, H. Zhong, Q. Liao, D. Huang, L. Hu, and Y. Guo, Improvement of self-referenced continuous-variable quantum key distribution with quantum photon catalysis, Opt. Express 27, 17186 (2019).

[43] E. V. Mikheev, A. S. Pugin, D. A. Kuts, S. A. Podoshvedov, and N. B. An, Efficient production of large-size optical schrödinger cat states, Scientific Reports 9, 14301 (2019)

[44] M. D. Eisaman, J. Fan, A. Migdall, and S. V. Polyakov, Invited review article: Single-photon sources and detectors, Review of Scientific Instruments 82, 071101 (2011) 
[45] I. Esmaeil Zadeh, J. Chang, J. W. N. Los, S. Gyger, A. W. Elshaari, S. Steinhauer, S. N. Dorenbos, and V. Zwiller, Superconducting nanowire single-photon detectors: A perspective on evolution, state-of-the-art, future developments, and applications, Applied Physics Letters 118, 190502 (2021), https://doi.org/10.1063/5.0045990.

[46] T. Günthner, B. Pressl, K. Laiho, J. Geßler, S. Höfling,
M. Kamp, C. Schneider, and G. Weihs, Broadband indistinguishability from bright parametric downconversion in a semiconductor waveguide, Journal of Optics 17, 125201 (2015)

[47] M. S. Kim, W. Son, V. Bužek, and P. L. Knight, Entanglement by a beam splitter: Nonclassicality as a prerequisite for entanglement, Phys. Rev. A 65, 032323 (2002) 